\title{
Correction to: Immunomodulatory Effects of CP-25 on Splenic T Cells of Rats with Adjuvant Arthritis
}

\author{
Yang Wang, ${ }^{1}$ Chen-chen Han, ${ }^{1}$ Dongqian Cui, ${ }^{1}$ Ting-ting Luo, ${ }^{1}$ Yifan Li, ${ }^{1}$ Yuwen Zhang, \\ Yang Ma, ${ }^{1,2}$ and Wei Wei ${ }^{1,2}$
}

Correction to: Inflammation (2018)

https://doi.org/10.1007/s10753-018-0757-z

The original version of this article contained mistakes, and the authors would like to correct them. The correct details are given below:

- In Results section under "CP-25 Downregulated GRK2 and EP4 Co-expression of Spleen Tissue in AA Rats" section, the citation "(Fig. 3c, d)" should be "(Fig. 3a, b)".

- In Results section, the heading "CP-25 Downregulated the Level of cAMP in $\mathrm{T}$ Cell of AA Rats" should be "CP-25 Upregulated the Level of cAMP in T Cell of AA Rats", thus, the sentence "whereas CP-25 significantly reduced cAMP levels

The online version of the original article can be found at https://doi.org/ 10.1007/s10753-018-0757-z

\footnotetext{
${ }^{1}$ Institute of Clinical Pharmacology, Anhui Medical University, Key Laboratory of Anti-inflammatory and Immune Medicine, Ministry of Education, Anhui Collaborative Innovation Center of Anti-inflammatory and Immune Medicine, Meishan Road 81, Hefei, 230032, China

${ }^{2}$ To whom correspondence should be addressed at Institute of Clinical Pharmacology, Anhui Medical University, Key Laboratory of Antiinflammatory and Immune Medicine, Ministry of Education, Anhui Collaborative Innovation Center of Anti-inflammatory and Immune Medicine, Meishan Road 81, Hefei, 230032, China. E-mails: mayang_ahmu@126.com; wwei@ahmu.edu.cn
}

in T cells to normal levels (Fig. 7)." should read as "whereas CP-25 significantly upregulated cAMP levels in T cells to normal levels (Fig. 7).".

- In Figure 7 caption, "Fig. 7. CP-25 downregulated T cell cAMP level in AA rats." should read as "Fig. 7. CP-25 upregulated T cell cAMP level in AA rats."

- In Conclusion section, the sentence "but also downregulated moderately inflammatory cytokines secreted by $\mathrm{T}$ cells during inflammation and cAMP levels in T cells." should read as "but also downregulated moderately inflammatory cytokines secreted by $\mathrm{T}$ cells during inflammation and upregulated cAMP levels in T cells.".

The authors apologize for potential issues raised by these mistakes. 